\title{
Parametric Vibrations of Axially Moving Beams with Multiple Edge Cracks
}

\author{
Murat Sarigül \\ Hafsa Sultan Mah. 4811 Sok. No:2/1 Yunusemre/Manisa, Turkey.
}

(Received 30 December 2015; accepted 31 January 2017)

Nonlinear transverse vibrations of axially moving beams with multiple cracks is handled studied. Assuming that the beam moves with mean velocity having harmonically variation, influence of the edge crack on the moving continua are investigated in this study. Due to existence of the crack in the transverse direction, the healthily beam is divided into parts. The translational and rotational springs are replaced between these parts so that high stressed regions around the crack tips are redefined with the springs' energies. Thus, the problem is converted to an axially moving spring-beam system. The equations of motion and its corresponding conditions are obtained by means of the Hamilton Principle. In numerical analysis, the natural frequencies and responses of the spring-beam system are investigated for principal parametric resonance in detail. Some important results are obtained; the natural frequencies decreases with increasing crack depth. In case of the beam travelling with high velocities, the effects of crack's depth on natural frequencies seems to be vanished.

\section{INTRODUCTION}

The studying of a crack problem for vibrating systems has been taking place in topic subjects. If a continuation consists of any crack, the stress near the crack tips merges to infinity. The effect of the crack on dynamic behavior of the beam was simulated by means of the fracture mechanics methods. When using these methods, generally two different approaches have been followed: local flexibility and stress/strain functions. Stress/strain functions are based on variation principle and decay exponentially. According to loading type and crack orientation on the beam, plate etc., the stress distributions near crack tips could be formulated by means of opening and shear mode Stress Intensity Factors (SIFs). Studies using these formulations could be summarized in brief: for cracked continua (beam, shaft, plate etc.), Dimarogonas summarized the studies done between years 1970-1993, ${ }^{1}$ Sih proposed strain energy density factor theory, ${ }^{2}$ and $\mathrm{Wu}$ studied maximum energy release rate criterion for a semi-infinite L-shaped crack. ${ }^{3}$ Tada et al. presented SIFs of different modes. ${ }^{4}$ Anifantis and Dimarogonas studied the system stability for the cracked column with vertical load. ${ }^{5}$ Christides and Barr presented an exponential-type crack disturbance function. ${ }^{6}$ Shen and Pierre proposed a similar approach for single-cracked beams. ${ }^{7}$ Chondros et al. studied the lateral vibration of simply supported beams. ${ }^{8}$ Yang et al. proposed an approach based on the stiffness definition of cracked beams. ${ }^{9}$ Mazanoglu et al. ${ }^{10}$ used the change of the strain energy distribution given by Yang et al. ${ }^{9}$ in order to analyze vibration of multiple-cracked non-uniform beams. Developing a new model based on displacement field, Behzad et al. studied simply supported beams with an edge crack. $^{11}$

For dynamics analysis of the cracked continua, springs have been used widely. By the definition of local flexibility around crack tip, cracked structures have been analyzed in several models that use a massless spring or reduced cross-section. Magnitudes of the local flexibility changes were estimated by the fracture mechanics methods. According to these methods, stress near the crack tip merges to infinity thus a singularity occurs at the crack tip. Presenting this case by SIFs, stresses in very small area were defined in terms of spring stiffness. When finding spring stiffness for vibration analysis of cracked structures, it is essential to have a load-deflection model for the entire body. When doing so, it is assumed that the cracked continuum consists of healthy parts connected by springs at the position of crack. The solution of equation or equation set was generally obtained by means of compatibility and continuity conditions at the crack location. For literature of cracked continua, following studies could be summarized in brief: Ostachowicz and Krawczuk ${ }^{12}$ developed a new local flexibility which was derived from the stress intensity factor by Anifantis and Dimarogonas. ${ }^{5}$ Taking into account the crack location and depth as well as the load, Sundermeyer et al. investigated a cracked beam. ${ }^{13}$ Yokoyama et al. studied beams having edge cracks of different depths at different positions. ${ }^{14}$ Wang et al. studied simple supported Euler-Bernoulli type beam with single edge open crack. ${ }^{15}$ Fernández-Sáez et al. studied fundamental frequencies of cracked Euler-Bernoulli beams. ${ }^{16}$ The effect of mass attachment on the free vibrations of cracked beams carrying a point mass has been discussed by Mermertas et al. ${ }^{17}$ By using the nonlinear spring model, Mendelshon et al. studied nonlinear free-vibration analysis of an Euler-Bernoulli beam with an edge crack. ${ }^{18}$ Considering coupling of bending and longitudinal vibration of the cracked cantilever beam, Rao et al. modeled the beam. ${ }^{19}$ Majkut studied the cantilever beam with an edge crack by using translational and rotational springs instead of the crack itself. ${ }^{20}$ The influence of location and depth of the crack on the first and second natural frequencies of flexural vibrations were analyzed. Loya et al. studied flexural vibrations of cracked micro- and nano-beams. ${ }^{21}$ By assuming the crossover flexibility constants to be too small, the crack was modeled with rotational and linear springs for clamped-clamped and simple-simple supporting cases.

Applying crack phenomenon to moving continua systems is a new research field. Therefore, it is essential not to mention too much of the background of studies done on moving 


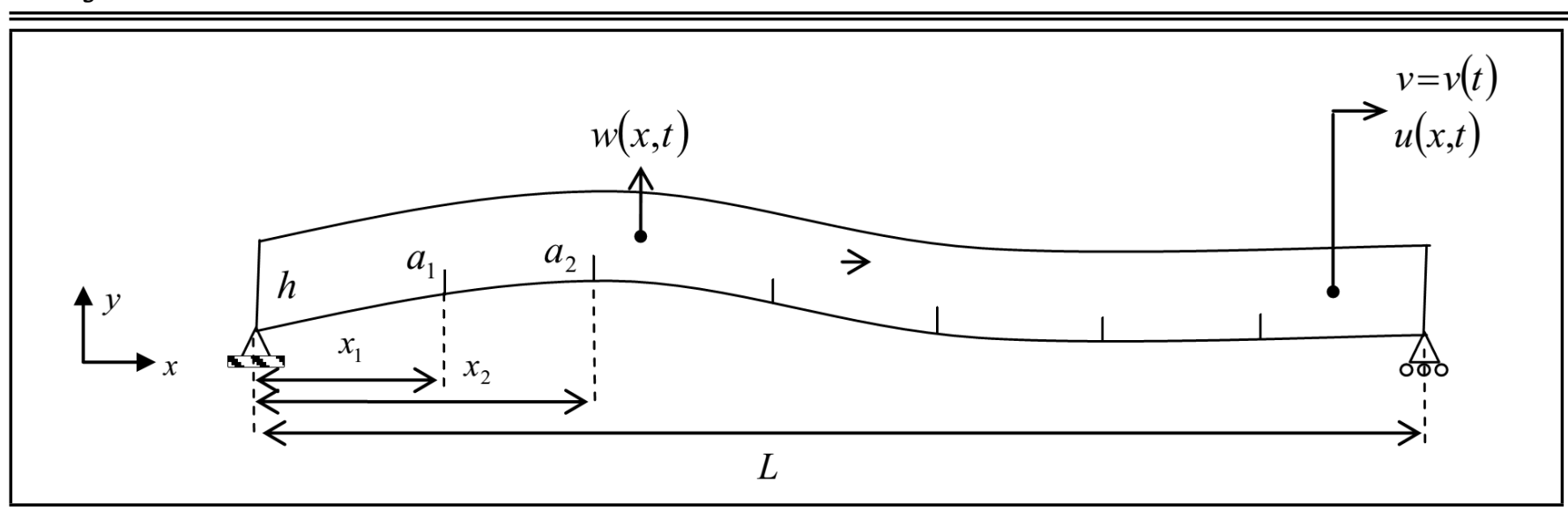

Figure 1. Axially moving beam with edge cracks.

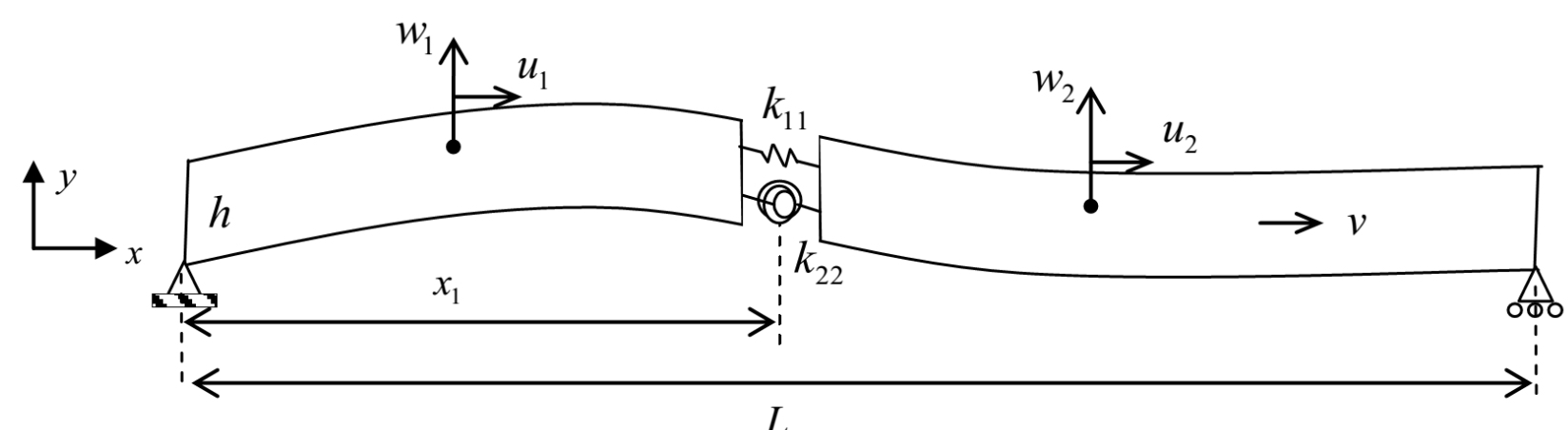

Figure 2. Spring-beam system.

continua. Moving continua's problem is resulted from beam's pre-stress, beam's axial velocity change, etc. Following studies could be summarized as a background to axially moving continua: when studying axially moving beam, generally transverse vibrations of it has been investigated. Simpson studied the beam in unstressed case. ${ }^{22}$ Pasin analyzed periodically reciprocating motion in axial direction, ${ }^{23}$ and Ponomareva and van Horssen studied it in stretched case. ${ }^{24}$ When solving axially moving beam problem, different solution methods have been used: the complex modal method ${ }^{25}$ and the variation method, ${ }^{26}$ the method of multiple scales, and the method of matched asymptotic expansions. ${ }^{27}$ Axially moving beams have been analyzed by subjecting it to a transverse force, ${ }^{28}$ by having small bending stiffness, ${ }^{29}$ and by considering multiple concentrated masses on the beam. ${ }^{30}$ The beam has been investigated under pinned-pinned supported case, ${ }^{31}$ under pinnedpinned and clamped-clamped supported cases $^{32}$ and while in touch with a small mass under pinned-pinned and clampedclamped boundary conditions. ${ }^{33}$ Vibrations of axially moving beam have been examined as continuously incoming and outgoing semi-infinite beam parts. ${ }^{34}$ When coming to vibration type, axially moving beams have been studied under planar vibration ${ }^{35}$ and nonlinear planar vibration. ${ }^{36}$ They've also been studied in the case of the coupled nonlinear resonant response $^{37}$ and coupling dynamics of transverse vibration and longitudinal vibration. ${ }^{38}$ The beam has been studied with assumption of parametric vibrations. ${ }^{39}$ Axially accelerating viscoelastic beams have been investigated under the combination and principal parametric resonances. ${ }^{40}$ In this study, vibrations of axially moving beams with multiple edge cracks have been investigated. For moving continua, cracks are assumed to be distributed with equal spans throughout the beam length. The beam moves with harmonically varying average transport velocity. A mathematical model of the continua has been built by using springs instead of the crack itself. By dividing the beam with $n$ cracks in number into $n+1$ parts and connecting them with springs, a beam-spring system has been constituted as a conjugate of cracked beam. The crack's influence on axially moving beam has been investigated according to the crack's position, height, as well as crack number. Damping factors and viscosity could be introduced when investigating nonlinear vibrations of the continua with crack.

\section{MODELLING OF THE PROBLEM}

An axially moving beam with multiple edge cracks could be drawn on $x-y$ domain as seen in Fig. 1. We've assumed that the beam having length $L$ consists of $n$ edge cracks in number. The cracks are located at an arbitrarily distance $x_{c}$ from simple support at left hand side. Cracks have different depths $a_{c}$ throughout beam's height $(h)$. We've assumed that the beam is of Euler-Bernoulli beam type. Under the assumption the beam moves with harmonically varying average transport velocity, $v=v(t)$, and transverse vibrations of the beam have been investigated.

For modeling continuum media (beam, plate, etc.) including cracks, one can assume that there are high stressed regions occurring around the crack tips when considering the whole system. These high stresses are resulted in stiffness reduction of the beam. To define these high stresses being concentrated at very small regions, translational, rotational, and interaction springs could be used in means of equivalence of the system. 


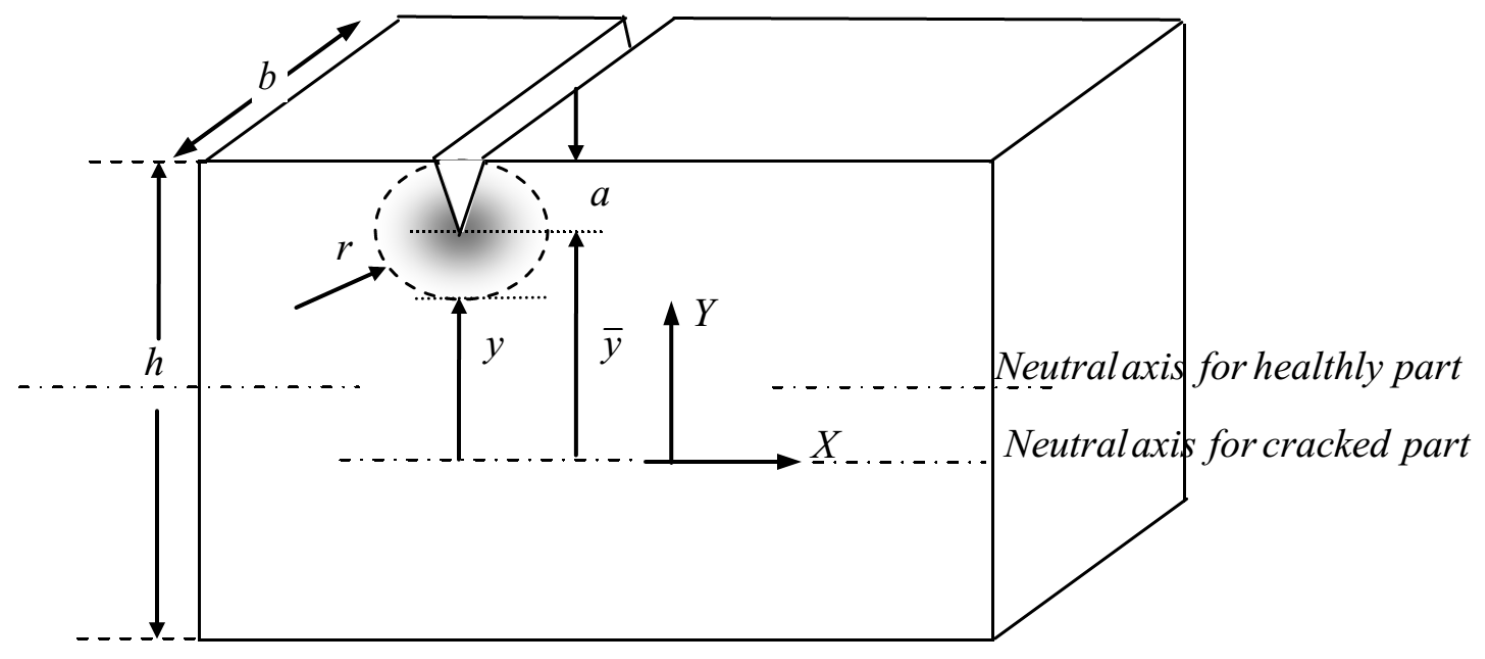

Figure 3. Geometry of a cracked beam

In other means, a cracked beam could be considered a springbeam system by means of statics. As seen in Fig. 2, the beam with one edge crack has been divided into two parts. These parts have been connected with each other by using springs. $w=w(x, t)$ is deflection of the beam, and $x_{1}$ is the crack location. The resulting system could be defined as spring-beam system. Using the rotational type spring $\left(k_{11}\right)$ is due to bending moment $(M)$. Using the translational spring $\left(k_{22}\right)$ is due to axial force $(F)$, which is resulted from axial stretching. For transverse vibrations of Euler-Bernoulli beam, effects of rotational and translational springs have been calculated separately. By using only two springs for one crack, it is easy to study the effect of one crack on transverse vibrations of axially moving beams. But when using Timoshenko beam theory, interaction between springs must be considered. This refers to a coupled case between axial force and bending moment.

\subsection{Finding Spring Constants}

In this study, an edge crack has been considered. Geometry of a cracked beam is seen in Fig. 3 .

In the light of strength of materials, ${ }^{41}$ normal stresses acting on the reduced cross section are given as

$$
\sigma_{x, w}=\frac{M y}{I_{c}} ; \quad \sigma_{x, u}=\frac{F}{A_{c}} ;
$$

where $M$ is bending moment, $F$ is axial force, $A_{c}=b(h-a)$ and $I_{c}=b(h-a)^{3} / 12$ are the cross-section and the second area inertial moment of the remaining part of the cracked beam, respectively. $a$ is crack's depth, $h$ and $b$ are width and height of the beam, respectively. Indexes $w$ and $u$ denote parameters related bending moment and axial force, respectively. The distance $y$ is found from Fig. 3 as

$$
\bar{y}=\frac{h-a}{2} \rightarrow y=\bar{y}-b .
$$

Singular stress distribution in fracture mechanics ${ }^{4}$ could be written in the following form:

$$
\sigma_{x, w}^{s}=\frac{K_{I, w}}{\sqrt{2 \pi r}} ; \quad \sigma_{x, u}^{s}=\frac{K_{I, u}}{\sqrt{2 \pi r}} ;
$$

where $r$ refers to the distance from the crack tip. $K_{I, w}$ and $K_{I, u}$ are opening mode Stress Intensify Factor (SIF) due to bending moment and axial force, respectively. If one browses the study done by Wang and Lee, it would be seen that the procedures we follow are the same. ${ }^{15}$ In this study, axial force has been considered in addition to bending moment.

Considering stress conditions as $\sigma_{x, w}^{s}=\sigma_{x, w}$ and $\sigma_{x, u}^{s}=$ $\sigma_{x, u}$ at the crack tips, the following expressions could be obtained:

$$
\begin{aligned}
& K_{I, w}=\sigma_{x, w}^{s} \sqrt{2 \pi r}=\frac{M}{I_{c}}\left(\frac{h-a}{2}-b_{w}\right) \sqrt{2 \pi b_{w}} \\
& K_{I, u}=\sigma_{x, u}^{s} \sqrt{2 \pi r}=\left(\frac{P}{A_{c}}\right) \sqrt{2 \pi b_{u}} .
\end{aligned}
$$

The distances $b_{w}$ and $b_{u}$ could be determined from the equilibrium conditions along the $x$-axis.

$$
\int_{0}^{b_{w}} \frac{K_{I, w}}{\sqrt{2 \pi r}} d r=\int_{\bar{y}-b_{w}}^{\bar{y}} \sigma_{x, w} d y ; \quad \int_{0}^{b_{w}} \frac{K_{I, u}}{\sqrt{2 \pi r}} d r=\int_{\bar{y}-b_{w}}^{\bar{y}} \sigma_{x, u} d y .
$$

By using Eq. (4), the left hand sides of Eq. (5) could be evaluated. In the same way, the evaluating the right hand sides of Eq. (5) by using Eq. (1) gives the following distances:

$$
b_{w}=\frac{h-a}{3} ; \quad b_{u}=\frac{h-a}{12} .
$$

Substituting Eq. (6) into Eq. (4), the SIFs could be expressed as

$$
K_{I, w}=\frac{6 M}{t h^{3 / 2}} F_{I, w}(a / h) ; \quad K_{I, u}=\frac{2 P}{t h^{1 / 2}} F_{I, u}(a / h) ;
$$

where

$$
F_{I, w}(a / h)=\frac{\sqrt{\frac{2}{27} \pi}}{(1-a / h)^{3 / 2}} ; \quad F_{I, u}(a / h)=\frac{\sqrt{\frac{2}{48} \pi}}{(1-a / h)^{1 / 2}} .
$$

See Wang and Lee for further information related with $K_{I, w} \cdot{ }^{15}$ Additionally, for cantilever beam, opening mode SIFs resulting from axial force and bending moment could be seen from the 
study presented by Majkut. ${ }^{20}$ SIFs in Eq. (7) could be written as

$$
K_{I}=K_{I, w}+K_{I, u} .
$$

Strain energy density function ${ }^{31,32,37}$ are given as

$$
J=\frac{K_{I}^{2}}{E^{\prime}}
$$

where $E^{\prime}=E /\left(1-\nu^{2}\right)$ for plane strain and $E$ and $\nu$ are Young's modulus and Poisson's ratio, respectively. By integrating the density function over the crack depth, the strain energy due to the crack is obtained. By applying Castiglione's theorem upon this energy, flexibilities could be written as

$$
\begin{aligned}
& c_{11}=\frac{\partial^{2}}{\partial M^{2}}\left[t \int_{0}^{a} J(a) d a\right] ; \\
& c_{22}=\frac{\partial^{2}}{\partial P^{2}}\left[t \int_{0}^{a} J(a) d a\right] ; \\
& c_{12}=c_{21}=\frac{\partial^{2}}{\partial M \partial P}\left[t \int_{0}^{a} J(a) d a\right] ;
\end{aligned}
$$

where $c_{11}$ is flexibility due to the bending moment, $c_{22}$ is due to the axial force, and $c_{12}=c_{21}$ is crossover flexibility resulting from interaction between axial force and bending moment. Loya et al. mentioned that the effects resulting from interactions between axial forces and bending moment could be ignored. ${ }^{21}$ By evaluating Eq. (11), flexibilities could be written in the following forms:

$$
c_{11}=\frac{L}{E^{\prime} I} \hat{c}_{11} ; \quad c_{22}=\frac{L}{E^{\prime} A} \hat{c}_{22} ;
$$

where $\hat{c}$ corresponds to non-dimensional flexibility and could be written as follows:

$$
\hat{c}_{11}=\frac{2 \pi}{9} \chi \frac{1-(1-\alpha)^{2}}{(1-\alpha)^{2}} ; \quad \hat{c}_{22}=\frac{2 \pi}{6} \chi \ln (1-\alpha) ;
$$

where $\alpha=\frac{a}{h}$ and $\chi=\frac{h}{L}$ are non-dimensional crack depth and slightness coefficient, respectively. Finally, spring constants could be obtained as follows:

$$
k_{11}=c_{11}^{-1} ; \quad k_{22}=c_{22}^{-1} .
$$

\subsection{Equations of Motion}

In this section energy approach has been used. Loya et al. presented increments of the energy due to spring and the crossover flexibility constants were assumed to be too small in their study. ${ }^{21}$ By ignoring the crossover flexibility, the increment of energy could be written as follows:

$$
\Delta U_{c}=\left.\left\{\frac{1}{2} k_{11} \Delta \theta^{2}+\frac{1}{2} k_{22} \Delta u^{2}\right\}\right|_{x=x_{c}} .
$$

$\Delta \theta$ is the angle rotated by the rotational spring; $\Delta u$ is the relative horizontal displacement at the edge crack section.

Let us assume that $n$ is the number of edge cracks on the beam. Modeling the problem by means of springs, it would be the same with axially moving beams carrying multiple concentrated masses. ${ }^{30}$ In order to derive the equations of motion of the beam-spring system, we use the Hamilton Principle as seen below:

$$
\begin{aligned}
& \delta \int_{t_{1}}^{t_{2}}\left\{\frac{1}{2} \sum_{m=0}^{n} \int_{x_{m}}^{x_{m+1}} \rho A\left(\sqrt{\left(\frac{d w_{m+1}}{d t}\right)^{2}+\left(\frac{d u_{m+1}}{d t}+v\right)^{2}}\right)^{2} d x-\right. \\
& \frac{1}{2} \sum_{m=0}^{n} \int_{x_{m}}^{x_{m+1}} E A\left(u_{m+1}^{\prime}+\frac{1}{2} w_{m+1}^{\prime 2}\right)^{2} d x- \\
& \frac{1}{2} \sum_{m=0}^{n} \int_{x_{m}}^{x_{m+1}} E I\left(w_{m+1}^{\prime \prime}\right)^{2} d x- \\
& \sum_{m=0}^{n}\left\{\frac{1}{2}\left(k_{11}\right)_{m}\left(w_{m+1}^{\prime}-w_{m}^{\prime}\right)^{2}+\right. \\
&\left.\left.\frac{1}{2}\left(k_{22}\right)_{m}\left(u_{m+1}-u_{m}\right)^{2}\right\}\left.\right|_{x=x_{m}}\right\} d t=0
\end{aligned}
$$

where first terms are kinetic energies of the beam. Other terms are of potential energy due to stretching, bending and springs. $v$ is velocity and prime denotes derivative to $x . w=w(x, t)$ and $u=u(x, t)$ are defined as transversal and longitudinal displacements, respectively. Other properties of the beam are such that, $\rho$ is the density, $A$ is the cross sectional area and $I$ is the moment of inertia of the beam cross-section with respect to the neutral axis.

Performing necessary calculations in Eq. (16), it was observed that the axial displacement could be eliminated from the equations. ${ }^{42}$ Finally, the following equations of motion and their corresponding conditions could be obtained:

$$
\begin{gathered}
\rho A\left(\frac{\partial^{2} w_{m+1}}{\partial t^{2}}+2 \frac{\partial^{2} w_{m+1}}{\partial x \partial t} v+\frac{\partial^{2} w_{m+1}}{\partial x^{2}} v^{2}+\frac{\partial v}{\partial t} \frac{\partial w_{m+1}}{\partial x}\right)+ \\
E I \frac{\partial^{4} w_{m+1}}{\partial x^{4}}=\frac{E A}{L}\left(1-\frac{1}{1+\frac{L}{E A}\left(k_{22}\right)_{p}}\right) \\
\left(\frac{1}{2} \sum_{r=0}^{n} \int_{x_{r}}^{x_{r+1}} \frac{\partial w_{r+1}^{2}}{\partial x} d x\right) \frac{\partial^{2} w_{m+1}}{\partial x^{2}} ; \quad(17 \mathbf{a}) \\
\left.w_{1}\right|_{x=x_{0}}=\left.w_{n+1}\right|^{x=x_{n+1}}=\left.E I \frac{\partial^{2} w_{1}}{\partial x^{2}}\right|_{x=x_{0}}= \\
-\left.E I \frac{\partial^{2} w_{n+1}}{\partial x^{2}}\right|^{x=x_{n+1}}=0
\end{gathered}
$$

$\left.w_{p}\right|_{x=x_{p}}=\left.w_{p+1}\right|_{x=x_{p}} ;$

$\left.E I \frac{\partial^{2} w_{p}}{\partial x^{2}}\right|_{x=x_{p}}=\left.E I \frac{\partial^{2} w_{p+1}}{\partial x^{2}}\right|_{x=x_{p}} ;$

$\left.E I \frac{\partial^{2} w_{p+1}}{\partial x^{2}}\right|_{x=x_{p}}=\left.\left(k_{11}\right)_{p}\left(\frac{\partial w_{p+1}}{\partial x}-\frac{\partial w_{p}}{\partial x}\right)\right|_{x=x_{p}} ;$

$\left.\left(E I \frac{\partial^{3} w_{p+1}}{\partial x^{3}}-E I \frac{\partial^{3} w_{p}}{\partial x^{3}}\right)\right|_{x=x_{p}}=$

$$
\frac{E A}{L}\left(1-\frac{1}{1+\frac{L}{E A}\left(k_{22}\right)_{p}}\right)\left(\frac{1}{2} \sum_{r=0}^{n} \int_{x_{r}}^{x_{r+1}}{\frac{\partial w_{r+1}}{\partial x}}^{2} d x\right) .
$$




$$
\left.\left(\frac{\partial w_{p+1}}{\partial x}-\frac{\partial w_{p}}{\partial x}\right)\right|_{x=x_{p}}
$$

In case of taking the crossover flexibility into account, matching conditions could be seen in Rao. ${ }^{19}$ From Eqs. (17a-17b), one would obtain the equations of motion and their corresponding matching conditions for the linear simply supported beam problem given in Wang and Lee under assumption that the axial velocity is zero. ${ }^{15}$ When obtaining differential equations in their non-dimensional forms, the following quantities have been used:

$$
\begin{aligned}
I & =A \kappa^{2} ; & \hat{w}(\hat{x}, \hat{t}) & =\frac{w(x, t)}{\kappa} ; & \hat{x} & =\frac{x}{L} ; \\
\eta_{p} & =\frac{x_{p}}{L} ; & \alpha_{p} & =\frac{a_{p}}{h} ; & \hat{v} & =\frac{\tau}{L} v ; \\
\hat{t} & =\frac{t}{\tau} ; & \tau & =\sqrt{\frac{\rho A L^{4}}{E I}} ; & v_{f}^{2} & =\frac{I}{A L^{2}} ;
\end{aligned}
$$

where $\kappa$ is radius of gyration for the beam's cross section. $\eta_{p}$ and $\alpha_{p}$ are defined as crack's position and depth, respectively. They are our control parameters.

$$
\begin{aligned}
& \left(\frac{\partial^{2} \hat{w}_{m+1}}{\partial \hat{t}^{2}}+2 \hat{v} \frac{\partial^{2} \hat{w}_{m+1}}{\partial \hat{x} \partial \hat{t}}+\frac{\partial^{2} \hat{w}_{m+1}}{\partial \hat{x}^{2}} \hat{v}^{2}+\frac{\partial \hat{v}}{\partial \hat{t}} \frac{\partial \hat{w}_{m+1}}{\partial \hat{x}}\right)+ \\
& v_{f}^{2} \frac{\partial^{4} \hat{w}_{m+1}}{\partial \hat{x}^{4}}=v_{f}^{2}\left(1-\frac{1}{1-1 /\left(\hat{c}_{22}\right)_{p}}\right) \text {. } \\
& \left(\frac{1}{2} \sum_{r=0}^{n} \int_{\hat{x}_{r}}^{\hat{x}_{r+1}} \frac{\partial \hat{w}_{r+1}^{2}}{\partial \hat{x}} d \hat{x}\right) \frac{\partial^{2} \hat{w}_{m+1}}{\partial \hat{x}^{2}} ; \\
& \left.\hat{w}_{1}\right|_{\hat{x}=\eta_{0}}=\left.\hat{w}_{n+1}\right|_{\hat{x}=\eta_{n+1}}=\left.\frac{\partial^{2} \hat{w}_{1}}{\partial x^{2}}\right|_{\hat{x}=\eta_{0}}= \\
& \left.\frac{\partial^{2} \hat{w}_{n+1}}{\partial x^{2}}\right|_{\hat{x}=\eta_{n+1}}=0 ; \\
& \left.\hat{w}_{p}\right|_{\hat{x}=\eta_{p}}=\left.\hat{w}_{p+1}\right|_{\hat{x}=\eta_{p}} ; \\
& \left.\frac{\partial^{2} \hat{w}_{1}}{\partial x^{2}}\right|_{\hat{x}=\eta_{p}}=\left.\frac{\partial^{2} \hat{w}_{p+1}}{\partial x^{2}}\right|_{\hat{x}=\eta_{p}} ; \\
& \left.\frac{\partial^{2} \hat{w}_{p+1}}{\partial \hat{x}^{2}}\right|_{\hat{x}=\eta_{p}}=\left.\frac{1}{\left(\hat{c}_{11}\right)_{p}}\left(\frac{\partial \hat{w}_{p+1}}{\partial \hat{x}}-\frac{\partial \hat{w}_{p}}{\partial \hat{x}}\right)\right|_{\hat{x}=\eta_{p}} ; \\
& \left.\left(\frac{\partial^{3} \hat{w}_{p+1}}{\partial \hat{x}^{3}}-\frac{\partial^{3} \hat{w}_{p}}{\partial \hat{x}^{3}}\right)\right|_{\hat{x}=\eta_{p}}= \\
& \left(1-\frac{1}{1-1 /\left(\hat{c}_{22}\right)_{p}}\right)\left(\frac{1}{2} \sum_{r=0}^{n} \int_{\hat{x}_{r}}^{\hat{x}_{r+1}} \frac{\partial \hat{w}_{r+1}^{2}}{\partial \hat{x}} d \hat{x}\right) . \\
& \left.\left(\frac{\partial \hat{w}_{p+1}}{\partial \hat{x}}-\frac{\partial \hat{w}_{p}}{\partial \hat{x}}\right)\right|_{\hat{x}=\eta_{p}} ;
\end{aligned}
$$

where $\eta_{0}=0, \eta_{n+1}=1, p=1 \ldots n$.

\section{ANALYTICAL SOLUTIONS: PERTURBATION ANALYSIS}

In this section, the approximate solutions are searched for the Eqs. (19a-19b). In order to tune these integral-partial differential equations and their corresponding conditions, we've used following treatment (see Öz et al. for details ${ }^{42}$ )

$$
\hat{w}_{m+1}(x, t ; \varepsilon)=\sqrt{\varepsilon} \tilde{w}_{m+1} .
$$

Substituting Eq. (20) into Eqs. (19a-19b) gives the following weakly nonlinear system:

$$
\begin{aligned}
& \frac{\partial^{2} \tilde{w}_{m+1}}{\partial \hat{t}^{2}}+2 \hat{v} \frac{\partial^{2} \tilde{w}_{m+1}}{\partial \hat{x} \partial \hat{t}}+\hat{v}^{2} \frac{\partial^{2} \tilde{w}_{m+1}}{\partial \hat{x}^{2}}+\frac{\partial \hat{v}}{\partial \hat{t}} \frac{\partial \tilde{w}_{m+1}}{\partial \hat{x}}+ \\
& v_{f}^{2} \frac{\partial^{4} \tilde{w}_{m+1}}{\partial \hat{x}^{4}}=\varepsilon v_{f}^{2}\left(1-\frac{1}{1-1 /\left(\hat{c}_{22}\right)_{p}}\right) . \\
& \left(\frac{1}{2} \sum_{r=0}^{n} \int_{\eta_{r}}^{\eta_{r+1}} \frac{\partial \tilde{w}_{r+1}^{2}}{\partial \hat{x}} d \hat{x}\right) \frac{\partial^{2} \tilde{w}_{m+1}}{\partial \hat{x}^{2}} ; \\
& \left.\tilde{w}_{1}\right|_{\hat{x}=\eta_{0}}=\left.\tilde{w}_{n+1}\right|_{\hat{x}=\eta_{n+1}}=\left.\frac{\partial^{2} \tilde{w}_{1}}{\partial x^{2}}\right|_{\hat{x}=\eta_{0}}= \\
& \left.\frac{\partial^{2} \tilde{w}_{n+1}}{\partial x^{2}}\right|_{\hat{x}=\eta_{n+1}}=0 \\
& \left.\tilde{w}_{p}\right|_{\hat{x}=\eta_{p}}=\left.\tilde{w}_{p+1}\right|_{\hat{x}=\eta_{p}} ; \\
& \left.\frac{\partial^{2} \tilde{w}_{1}}{\partial x^{2}}\right|_{\hat{x}=\eta_{p}}=\left.\frac{\partial^{2} \tilde{w}_{p+1}}{\partial x^{2}}\right|_{\hat{x}=\eta_{p}} ; \\
& \left.\frac{\partial^{2} \tilde{w}_{p+1}}{\partial \hat{x}^{2}}\right|_{\hat{x}=\eta_{p}}=\left.\frac{1}{\left(\hat{c}_{11}\right)_{p}}\left(\frac{\partial \tilde{w}_{p+1}}{\partial \hat{x}}-\frac{\partial \tilde{w}_{p}}{\partial \hat{x}}\right)\right|_{\hat{x}=\eta_{p}} ; \\
& \left.\left(\frac{\partial^{3} \tilde{w}_{p+1}}{\partial \hat{x}^{3}}-\frac{\partial^{3} \tilde{w}_{p}}{\partial \hat{x}^{3}}\right)\right|_{\hat{x}=\eta_{p}}= \\
& \varepsilon\left(1-\frac{1}{1-1 /\left(\hat{c}_{22}\right)_{p}}\right)\left(\frac{1}{2} \sum_{r=0}^{n} \int_{\eta_{r}}^{\eta_{r+1}} \frac{\partial \tilde{w}_{r+1}^{2}}{\partial \hat{x}} d \hat{x}\right) . \\
& \left.\left(\frac{\partial \tilde{w}_{p+1}}{\partial \hat{x}}-\frac{\partial \tilde{w}_{p}}{\partial \hat{x}}\right)\right|_{\hat{x}=\eta_{p}} .
\end{aligned}
$$

In order to find approximate solutions, we've applied the Method of Multiple Scales (MMS). We've assumed expansions of the forms

$$
\tilde{w}_{m+1}(x, t ; \varepsilon)=\tilde{w}_{1(m+1)}\left(x, T_{0}, T_{1}\right)+\varepsilon \tilde{w}_{2(m+1)}\left(x, T_{0}, T_{1}\right) ;
$$

where $\varepsilon$ is the book-keeping parameter artificially inserted into the equations. $T_{0}=t$ is fast time scale, and $T_{1}=\varepsilon t$ is slow time scale. Time derivatives are written as

$$
\frac{d}{d t}=D_{0}+\varepsilon D_{1}+\ldots ; \quad \frac{d^{2}}{d t^{2}}=D_{0}^{2}+2 \varepsilon D_{0} D_{1}+\ldots ;
$$

where $D_{0}$ and $D_{1}$ indicate the time derivatives with respect to $T_{0}$ and $T_{1}$, respectively.

Let us consider the velocity of the beam varies harmonically about a constant velocity $\hat{v}_{0}$ as follows:

$$
\hat{v}=\hat{v}_{0}+\varepsilon \hat{v}_{1} \sin (\Omega t)
$$

$\varepsilon \hat{v}_{1}$ and $\Omega$ is the magnitude and frequency of the harmonically variation, respectively. Inserting Eqs. (22-24) into Eqs. (21a$21 \mathrm{~b}$ ), and equating like powers of $\varepsilon$, and omitting the higher terms of $\varepsilon$ in the resulting equations, one obtains a general form for any power of $\varepsilon$ as follows:

order $\varepsilon(0)$

$D_{0}^{2} \tilde{w}_{1(m+1)}+2 \hat{v}_{0} D_{0} \frac{\partial \tilde{w}_{1(m+1)}}{\partial \hat{x}}+\hat{v}_{0}^{2} \frac{\partial^{2} \tilde{w}_{1(m+1)}}{\partial \hat{x}^{2}}+$ 


$$
\begin{aligned}
& v_{f}^{2} \frac{\partial^{4} \tilde{w}_{1(m+1)}}{\partial \hat{x}^{4}}=0 \\
& \left.\tilde{w}_{11}\right|_{\hat{x}=\eta_{0}}=\left.\tilde{w}_{1(n+1)}\right|_{\hat{x}=\eta_{n+1}}=\left.\frac{\partial^{2} \tilde{w}_{11}}{\partial x^{2}}\right|_{\hat{x}=\eta_{0}}= \\
& \left.\frac{\partial^{2} \tilde{w}_{1(n+1)}}{\partial x^{2}}\right|_{\hat{x}=\eta_{n+1}}=0 ; \\
& \left.\tilde{w}_{1 p}\right|_{\hat{x}=\eta_{p}}=\left.\tilde{w}_{1(p+1)}\right|_{\hat{x}=\eta_{p}} ; \\
& \left.\frac{\partial^{2} \tilde{w}_{1 p}}{\partial x^{2}}\right|_{\hat{x}=\eta_{p}}=\left.\frac{\partial^{2} \tilde{w}_{1(p+1)}}{\partial x^{2}}\right|_{\hat{x}=\eta_{p}} ; \\
& \left.\frac{\partial^{3} \tilde{w}_{1 p}}{\partial x^{3}}\right|_{\hat{x}=\eta_{p}}=\left.\frac{\partial^{3} \tilde{w}_{1(p+1)}}{\partial x^{3}}\right|_{\hat{x}=\eta_{p}} ; \\
& \left.\frac{\partial^{2} \tilde{w}_{1(p+1)}}{\partial \hat{x}^{2}}\right|_{\hat{x}=\eta_{p}}=\left.\frac{1}{\left(\hat{c}_{11}\right)_{p}}\left(\frac{\partial \tilde{w}_{1(p+1)}}{\partial \hat{x}}-\frac{\partial \tilde{w}_{1 p}}{\partial \hat{x}}\right)\right|_{\hat{x}=\eta_{p}} ; \\
& \left.\left(\frac{\partial^{3} \tilde{w}_{1(p+1)}}{\partial \hat{x}^{3}}-\frac{\partial^{3} \tilde{w}_{1 p}}{\partial \hat{x}^{3}}\right)\right|_{\hat{x}=\eta_{p}}=0 \\
& \text { order } \varepsilon(1) \\
& D_{0}^{2} \tilde{w}_{2(m+1)}+2 \hat{v} D_{0} \frac{\partial \tilde{w}_{2(m+1)}}{\partial \hat{x}}+\hat{v}^{2} \frac{\partial^{2} \tilde{w}_{2(m+1)}}{\partial \hat{x}^{2}}+ \\
& v_{f}^{2} \frac{\partial^{4} \tilde{w}_{2(m+1)}}{\partial \hat{x}^{4}}=-\left(2 \hat{v}_{0} \hat{v}_{1} \sin (\Omega t) \frac{\partial^{2} \tilde{w}_{1(m+1)}}{\partial \hat{x}^{2}}+\right. \\
& \begin{array}{l}
\left.\frac{\partial^{2} Y_{n+1}}{\partial x^{2}}\right|_{\hat{x}=\eta_{n+1}}=0 \\
\left.Y_{p}\right|_{\hat{x}=\eta_{p}}=\left.Y_{p+1}\right|_{\hat{x}=\eta_{p}}
\end{array} \\
& \left.\frac{\partial^{2} Y_{p}}{\partial x^{2}}\right|_{\hat{x}=\eta_{p}}=\left.\frac{\partial^{2} Y_{p+1}}{\partial x^{2}}\right|_{\hat{x}=\eta_{p}} ; \\
& \left.\left(\frac{\partial Y_{p+1}}{\partial \hat{x}}-\frac{\partial Y_{p}}{\partial \hat{x}}\right)\right|_{\hat{x}=\eta_{p}}=\left.\left(\hat{c}_{11}\right)_{p} \frac{\partial^{2} Y_{p+1}}{\partial \hat{x}^{2}}\right|_{\hat{x}=\eta_{p}} ; \\
& \left.\left(\frac{\partial^{3} Y_{p+1}}{\partial \hat{x}^{3}}-\frac{\partial^{3} Y_{p}}{\partial \hat{x}^{3}}\right)\right|_{\hat{x}=\eta_{p}}=0 \\
& v_{f}^{2} \frac{\partial^{4} Y_{m+1}}{\partial \hat{x}^{4}}+\hat{v}_{0}^{2} \frac{\partial^{2} Y_{m+1}}{\partial \hat{x}^{2}}+2 i \hat{v}_{0} \omega \frac{\partial Y_{m+1}}{\partial \hat{x}}-\omega^{2} Y_{m+1}=0 ; \\
& \left.Y_{1}\right|_{\hat{x}=\eta_{0}}=\left.Y_{n+1}\right|_{\hat{x}=\eta_{n+1}}=\left.\frac{\partial^{2} Y_{1}}{\partial x^{2}}\right|_{\hat{x}=\eta_{0}}= \\
& \begin{array}{l}
\left.\frac{\partial^{2} Y_{n+1}}{\partial x^{2}}\right|_{\hat{x}=\eta_{n+1}}=0 ; \\
\left.Y_{p}\right|_{\hat{x}=\eta_{p}}=\left.Y_{p+1}\right|_{\hat{x}=\eta_{p}} ;
\end{array}
\end{aligned}
$$$$
\hat{v}_{1} \Omega \cos (\Omega t) \frac{\partial \tilde{w}_{1(m+1)}}{\partial \hat{x}}+2 \hat{v}_{1} D_{0} \frac{\partial \tilde{w}_{1(m+1)}}{\partial \hat{x}}+
$$$$
\left.2 \hat{v}_{0} D_{1} \frac{\partial \tilde{w}_{1(m+1)}}{\partial \hat{x}}+2 D_{0} D_{1} \tilde{w}_{1(m+1)}\right)+
$$$$
v_{f}^{2}\left(1-\frac{1}{1-1 /\left(\hat{c}_{22}\right)_{p}}\right)\left(\frac{1}{2} \sum_{r=0}^{n} \int_{\eta_{r}}^{\eta_{r+1}} \frac{\partial \tilde{w}_{1(r+1)}^{2}}{\partial \hat{x}} d \hat{x}\right) .
$$$$
\frac{\partial^{2} \tilde{w}_{1(m+1)}}{\partial \hat{x}^{2}}
$$

Solution of the eigenfunction $Y_{m+1}(x)$ can be written as follows:

$$
\begin{aligned}
Y_{m+1}(\hat{x})= & d_{(m+1) 1} e^{i \beta_{(m+1) 1} \hat{x}}+d_{(m+1) 2} e^{i \beta_{(m+1) 2} \hat{x}}+ \\
& d_{(m+1) 3} e^{i \beta_{(m+1) 3} \hat{x}}+d_{(m+1) 4} e^{i \beta_{(m+1) 4} \hat{x}}
\end{aligned}
$$

where $d_{(p+1) j}(j=1 \ldots 4)$ are constants, and are four roots of the following equation obtained from Eq. (29):

$$
v_{f}^{2} \beta_{m+1}^{4}+\hat{v}_{0}^{2} \beta_{m+1}^{2}-2 \hat{v}_{0} \omega \beta_{m+1}-\omega^{2}=0 .
$$

Second order $\varepsilon(1)$ in Eqs. (26a-26b) corresponds to nonlinear problem. Inserting Eq. (27) in the second order gives the following equations:

$$
\begin{aligned}
& D_{0}^{2} \tilde{w}_{2(m+1)}+2 \hat{v}_{0} D_{0} \frac{\partial \tilde{w}_{2(m+1)}}{\partial \hat{x}}+\hat{v}_{0}^{2} \frac{\partial^{2} \tilde{w}_{2(m+1)}}{\partial \hat{x}^{2}}+ \\
& v_{f}^{2} \frac{\partial^{4} \tilde{w}_{2(m+1)}}{\partial \hat{x}^{4}}=-\left(-\hat{v}_{0} \hat{v}_{1} i A e^{i(\Omega+\omega) T_{0}} \frac{\partial^{2} Y_{m+1}}{\partial \hat{x}^{2}}-\right. \\
& \left.\hat{v}_{0} \hat{v}_{1} i \bar{A} e^{i(\Omega-\omega) T_{0}} \frac{\partial^{2} \bar{Y}_{m+1}}{\partial \hat{x}^{2}}\right)- \\
& \left(\hat{v}_{0} \hat{v}_{1} i A e^{-i(\Omega-\omega) T_{0}} \frac{\partial^{2} Y_{m+1}}{\partial \hat{x}^{2}}+\right. \\
& \left.\hat{v}_{0} \hat{v}_{1} i \bar{A} e^{-i(\Omega+\omega) T_{0}} \frac{\partial^{2} \bar{Y}_{m+1}}{\partial \hat{x}^{2}}\right)- \\
& \left(\frac{1}{2} \hat{v}_{1} \Omega A e^{i(\Omega+\omega) T_{0}} \frac{\partial Y_{m+1}}{\partial \hat{x}}+\frac{1}{2} \hat{v}_{1} \Omega \bar{A} e^{i(\Omega-\omega) T_{0}} \frac{\partial \bar{Y}_{m+1}}{\partial \hat{x}}\right)- \\
& 2 \hat{v}_{1} i \omega A e^{i \omega T_{0}} \frac{\partial Y_{m+1}}{\partial \hat{x}}-\left(\frac{1}{2} \hat{v}_{1} \Omega A e^{-i(\Omega-\omega) T_{0}} \frac{\partial Y_{m+1}}{\partial \hat{x}}+\right.
\end{aligned}
$$$$
\left.\frac{1}{2} \hat{v}_{1} \Omega \bar{A} e^{-i(\Omega+\omega) T_{0}} \frac{\partial \bar{Y}_{m+1}}{\partial \hat{x}}\right)+2 \hat{v}_{1} i \omega \bar{A} e^{-i \omega T_{0}} \frac{\partial \bar{Y}_{m+1}}{\partial \hat{x}}+
$$$$
v_{f}^{2}\left(1-\frac{1}{1-1 /\left(\hat{c}_{22}\right)_{p}}\right) \text {. }
$$

Differential equations corresponding to order $\varepsilon(0)$ could be defined as a linear problem. For these equations with two variables, we assume the following solution form,

$$
\hat{w}_{1(m+1)}\left(\hat{x}, T_{0}, T_{1}\right)=A\left(T_{1}\right) e^{i \omega T_{0}} Y_{m+1}(\hat{x})+
$$




$$
\begin{aligned}
& \begin{array}{l}
\hline \frac{1}{2} A^{3} e^{3 i \omega T_{0}}\left(\sum_{r=0}^{n} \int_{\eta_{r}}^{\eta_{r+1}} \frac{\partial Y_{r+1}^{2}}{\partial \hat{x}} d \hat{x}\right)+ \\
\frac{1}{2} \bar{A}^{2} A e^{-i \omega T_{0}}\left(\sum_{r=0}^{n} \int_{\eta_{r}}^{\eta_{r+1}} \frac{\partial \bar{Y}_{r+1}^{2}}{\partial \hat{x}} d \hat{x}\right)+ \\
\left.A^{2} \bar{A} e^{i \omega T_{0}}\left(\sum_{r=0}^{n} \int_{\eta_{r}}^{\eta_{r+1}} \frac{\partial Y_{r+1}}{\partial \hat{x}} \frac{\partial \bar{Y}_{r+1}}{\partial \hat{x}} d \hat{x}\right)\right\} \frac{\partial^{2} Y_{m+1}}{\partial \hat{x}^{2}}-
\end{array} \\
& \frac{1}{2} \bar{A}^{3} e^{-3 i \omega T_{0}}\left(\sum_{r=0}^{n} \int_{\eta_{r}}^{\eta_{r+1}} \frac{\partial \bar{Y}_{r+1}^{2}}{\partial \hat{x}} d \hat{x}\right)+ \\
& \left.\frac{1}{2} A^{2} \bar{A} e^{i \omega T_{0}}\left(\sum_{r=0}^{n} \int_{\eta_{r}}^{\eta_{r+1}}{\frac{\partial Y_{r+1}}{\partial \hat{x}}}^{2} d \hat{x}\right)\right\} \\
& \left.\left(\frac{\partial \bar{Y}_{p+1}}{\partial \hat{x}}-\frac{\partial \bar{Y}_{p}}{\partial \hat{x}}\right)\right|_{\hat{x}=\eta_{p}} . \\
& \Omega=2 \omega+\varepsilon \sigma . \\
& \tilde{w}_{2(m+1)}=e^{i \omega T_{0}} \vartheta_{m+1}+W_{m+1}+c c ; \\
& -\omega^{2} \vartheta_{m+1}+2 i \omega \hat{v}_{0} \frac{\partial \vartheta_{m+1}}{\partial \hat{x}}+\hat{v}_{0}^{2} \frac{\partial^{2} \vartheta_{m+1}}{\partial \hat{x}^{2}}+v_{f}^{2} \frac{\partial^{4} \vartheta_{m+1}}{\partial \hat{x}^{4}}= \\
& -\left\{D_{1} A\left(2 \omega \hat{v}_{0} \frac{\partial Y_{m+1}}{\partial \hat{x}}+2 i \omega Y_{m+1}\right)+2 \hat{v}_{1} i \omega A \frac{\partial Y_{m+1}}{\partial \hat{x}}+\right. \\
& \bar{A} e^{i \sigma T_{1}} \hat{v}_{1}\left(-\hat{v}_{0} i \frac{\partial^{2} \bar{Y}_{m+1}}{\partial \hat{x}^{2}}+\omega \frac{\partial \bar{Y}_{m+1}}{\partial \hat{x}}\right)- \\
& A^{2} \bar{A} v_{f}^{2}\left(1-\frac{1}{1-1 /\left(\hat{c}_{22}\right)_{p}}\right) \text {. } \\
& {\left[\left(\sum_{r=0}^{n} \int_{\eta_{r}}^{\eta_{r+1}} \frac{\partial Y_{r+1}}{\partial \hat{x}} \frac{\partial \bar{Y}_{r+1}}{\partial \hat{x}} d \hat{x}\right) \frac{\partial^{2} Y_{m+1}}{\partial \hat{x}^{2}}+\right.} \\
& \left.\left.\frac{1}{2}\left(\sum_{r=0}^{n} \int_{\eta_{r}}^{\eta_{r+1}} \frac{\partial Y_{r+1}^{2}}{\partial \hat{x}} d \hat{x}\right) \frac{\partial^{2} \bar{Y}_{m+1}}{\partial \hat{x}^{2}}\right]\right\} \\
& \left.\vartheta_{1}\right|_{\hat{x}=\eta_{0}}=\left.\vartheta_{n+1}\right|_{\hat{x}=\eta_{n+1}}=\left.\frac{\partial^{2} \vartheta_{1}}{\partial x^{2}}\right|_{\hat{x}=\eta_{0}}= \\
& \left.\frac{\partial^{2} \vartheta_{n+1}}{\partial x^{2}}\right|_{\hat{x}=\eta_{n+1}}=0 \\
& \left.\vartheta_{p}\right|_{\hat{x}=\eta_{p}}=\left.\vartheta_{p+1}\right|_{\hat{x}=\eta_{p}} ; \\
& \left.\frac{\partial^{2} \vartheta_{p}}{\partial x^{2}}\right|_{\hat{x}=\eta_{p}}=\left.\frac{\partial^{2} \vartheta_{p+1}}{\partial x^{2}}\right|_{\hat{x}=\eta_{p}} \\
& \left.\left(\frac{\partial \vartheta_{p+1}}{\partial \hat{x}}-\frac{\partial \vartheta_{p}}{\partial \hat{x}}\right)\right|_{\hat{x}=\eta_{p}}=\left.\left(\hat{c}_{11}\right)_{p} \frac{\partial^{2} \vartheta_{p+1}}{\partial \hat{x}^{2}}\right|_{\hat{x}=\eta_{p}} ; \\
& \left.\left(\frac{\partial^{3} \vartheta_{p+1}}{\partial \hat{x}^{3}}-\frac{\partial^{3} \vartheta_{p}}{\partial \hat{x}^{3}}\right)\right|_{\hat{x}=\eta_{p}}=A^{2} \bar{A}\left(1-\frac{1}{1-1 /\left(\hat{c}_{22}\right)_{p}}\right) . \\
& \left\{\left.\left(\sum_{r=0}^{n} \int_{\eta_{r}}^{\eta_{r+1}} \frac{\partial Y_{r+1}}{\partial \hat{x}} \frac{\partial \bar{Y}_{r+1}}{\partial \hat{x}} d \hat{x}\right)\left(\frac{\partial Y_{p+1}}{\partial \hat{x}}-\frac{\partial Y_{p}}{\partial \hat{x}}\right)\right|_{\hat{x}=\eta_{p}}+\right.
\end{aligned}
$$

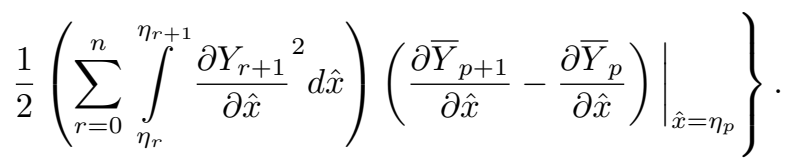


According to MMS, a solvability condition must be found from the last order. ${ }^{43}$ Invoking necessary calculations to find solvability conditions and doing some simplifications, the following could be obtained:

$$
K_{1} D_{1} A+K_{2} A+K_{3} A^{2} \bar{A}+K_{4} \bar{A} e^{i \sigma T_{1}}=0 ;
$$

where

$$
\begin{aligned}
& K_{1}=\sum_{r=0}^{n} \int_{\eta_{r}}^{\eta_{r+1}} 2 \bar{Y}_{r+1}\left(\hat{v}_{0} \frac{\partial Y_{r+1}}{\partial \hat{x}}+i \omega Y_{r+1}\right) d \hat{x} ; \\
& K_{2}=2 \hat{v}_{1} i \omega \sum_{r=0}^{n} \int_{\eta_{r}}^{\eta_{r+1}} \bar{Y}_{r+1} \frac{\partial Y_{r+1}}{\partial \hat{x}} d \hat{x} \\
& K_{3}=v_{f}^{2} \sum_{r=0}^{n}\left(1-\frac{1}{1-1 /\left(\hat{c}_{22}\right)_{p}}\right) \text {. } \\
& \left\{\left(\frac{1}{2}+\sum_{r=0}^{n} \int_{\eta_{r}}^{\eta_{r+1}} \frac{\partial Y_{r+1}}{\partial \hat{x}} \frac{\partial \bar{Y}_{r+1}}{\partial \hat{x}} d \hat{x}\right) .\right. \\
& \left(\sum_{r=0}^{n} \int_{\eta_{r}}^{\eta_{r+1}} \bar{Y}_{r+1} \frac{\partial^{2} Y_{r+1}}{\partial \hat{x}^{2}} d \hat{x}\right)+ \\
& {\left[\left(\sum_{r=0}^{n} \int_{\eta_{r}}^{\eta_{r+1}} \frac{\partial Y_{r+1}}{\partial \hat{x}} \frac{\partial \bar{Y}_{r+1}}{\partial \hat{x}} d \hat{x}\right)\left(\frac{\partial Y_{r+1}}{\partial \hat{x}}-\frac{\partial Y_{r}}{\partial \hat{x}}\right)+\right.} \\
& \left.\left.\frac{1}{2}\left(\frac{\partial \bar{Y}_{r+1}}{\partial \hat{x}}-\frac{\partial \bar{Y}_{r}}{\partial \hat{x}}\right)\right]\left.\bar{Y}_{r}\right|_{\hat{x}=\eta_{r}}\right\} ; \\
& K_{4}=\hat{v}_{1} \sum_{r=0}^{n} \int_{\eta_{r}}^{\eta_{r+1}} \bar{Y}_{r+1}\left(-\hat{v}_{0} i \frac{\partial^{2} Y_{r+1}}{\partial \hat{x}^{2}}+\omega \frac{\partial \bar{Y}_{r+1}}{\partial \hat{x}}\right) d \hat{x} \text {; } \\
& \sum_{r=0}^{n} \int_{\eta_{r}}^{\eta_{r+1}} \frac{\partial Y_{r+1}^{2}}{\partial \hat{x}} d \hat{x}=1
\end{aligned}
$$

The polar form is introduced as

$$
A=\frac{1}{2} a e^{i \theta} ; \quad \theta=\theta\left(T_{1}\right) .
$$

Inserting the polar form into Eq. (35), and separating real and imaginary parts, the following equations could be obtained:

$$
\begin{aligned}
K_{1}^{r e} \dot{a}+ & K_{1}^{i m}\left(\frac{1}{2} \sigma-\dot{\theta}\right) a+\left[K_{2}^{r e} a+\frac{1}{4} K_{3}^{r e} a^{3}\right]= \\
- & {\left[K_{4}^{r e} a \cos (2 \gamma)-K_{4}^{i m} a \sin (2 \gamma)\right] } \\
K_{1}^{i m} \dot{a}- & K_{1}^{r e}\left(\frac{1}{2} \sigma-\dot{\theta}\right) a+\left[K_{2}^{i m} a+\frac{1}{4} K_{3}^{i m} a^{3}\right]= \\
- & {\left[K_{4}^{i m} a \cos (2 \gamma)+K_{4}^{r e} a \sin (2 \gamma)\right] }
\end{aligned}
$$

where $\gamma=\sigma T_{1} / 2-\theta . a$ is real amplitude and $\theta$ is the phase. $r e$ and $i m$ indexes correspond to real and imaginary parts, respectively. By eliminating $\gamma$ in these equations, equations could be defined phase-modulation equations.

When analyzing the parametric vibrations, it could be assumed that the system is under the steady-state case during vibrations. $\dot{a}$ and $\dot{\theta}$ in Eq. (38) vanish and are taken as zero denoting no change in amplitude and phase with time. In this case, $a=a_{0}$ is a constant and fixed-points could be found. From Eq. (38), it could be seen that there is one trivial solution as $a_{0}=0$. For non-trivial solutions, following relation between the detuning parameter $(\sigma)$ and the amplitude could be derived by doing further simplification in Eq. (38):

$$
\begin{aligned}
& \left(\frac{1}{2} K_{1}^{i m} \sigma+\left[K_{2}^{r e}+\frac{1}{4} K_{3}^{r e} a_{0}^{2}\right]\right)^{2}+ \\
& \left(-\frac{1}{2} K_{1}^{r e} \sigma+\left[K_{2}^{i m}+\frac{1}{4} K_{3}^{i m} a_{0}^{2}\right]\right)^{2}=\left[K_{4}^{r e 2}+K_{4}^{i m^{2}}\right]
\end{aligned}
$$

$\sigma$ and $a_{0}$ could be simultaneously calculated by means of Eq. (39).

\section{RESULTS AND DISCUSSION}

Natural frequencies can be calculated numerically by using Eqs. (27-30). In order to understand well the effects of the axial velocity as well as geometrical parameters; crack's depth $(\alpha)$, and crack's position $(\eta)$ on the natural frequencies, these parameters have been drawn on the same graph. For case of one crack, the index $p$ was removed. By assigning $\chi=1 / 10$, $v_{f}=0.8$, natural frequency-mean axial velocity $\left(v_{0}\right)$ curves drawn in Figs. 4 through 6. First and second modes have been presented separately in these figures. For every case, it is seen that frequencies of the first and second modes decrease with increasing velocity.

In Fig. 4, natural frequency versus axial velocity has been drawn for different crack's depths, $(\alpha ; 0.1,0.5,0.7)$ when $\eta=0.5$. From these curves, natural frequencies would become at low values if the crack's depth is large (0.7). In other means, the beam's stiffness decreases with increasing crack's depth (0.7). Therefore, it was expected low natural frequencies for big cracks. For the first mode in Fig. 4a, natural frequencies decrease with increasing velocity and approach each other for different crack depths. This is result of small amplitude vibrations at high velocities. For the second mode in Fig. 4b, slow velocities have not any influence on natural frequencies when comparing the crack depths. Increasing velocity affects the symmetry of the second mode. Therefore, it is expected that beam stiffness would show its effect due to larger crack $(0.7)$ as velocity increases.

In Fig. 5, axial velocity versus natural frequency has been drawn for the different crack's position $(\eta ; 0.1,0.5)$, when $\alpha=0.3$. It could be seen from Fig. 5a that natural frequencies are low values as the crack takes place at the middle point of the beam instead of being near end supports. Thus, the stiffness's reduction of the beam due to the crack is effective on natural frequencies when crack takes place in the middle of the beam. This influence vanishes as velocity increases. For the second mode in Fig. 5b, it could be observed that the crack replaced in the middle of the beam does not have too much influence at low velocities. This is related with bending moment in case of second mode's symmetry. As velocity increases, the symmetry will vanish, then second mode will become first mode at a suitable velocity. See Sarigul and Boyaci ${ }^{30}$ for further details. 


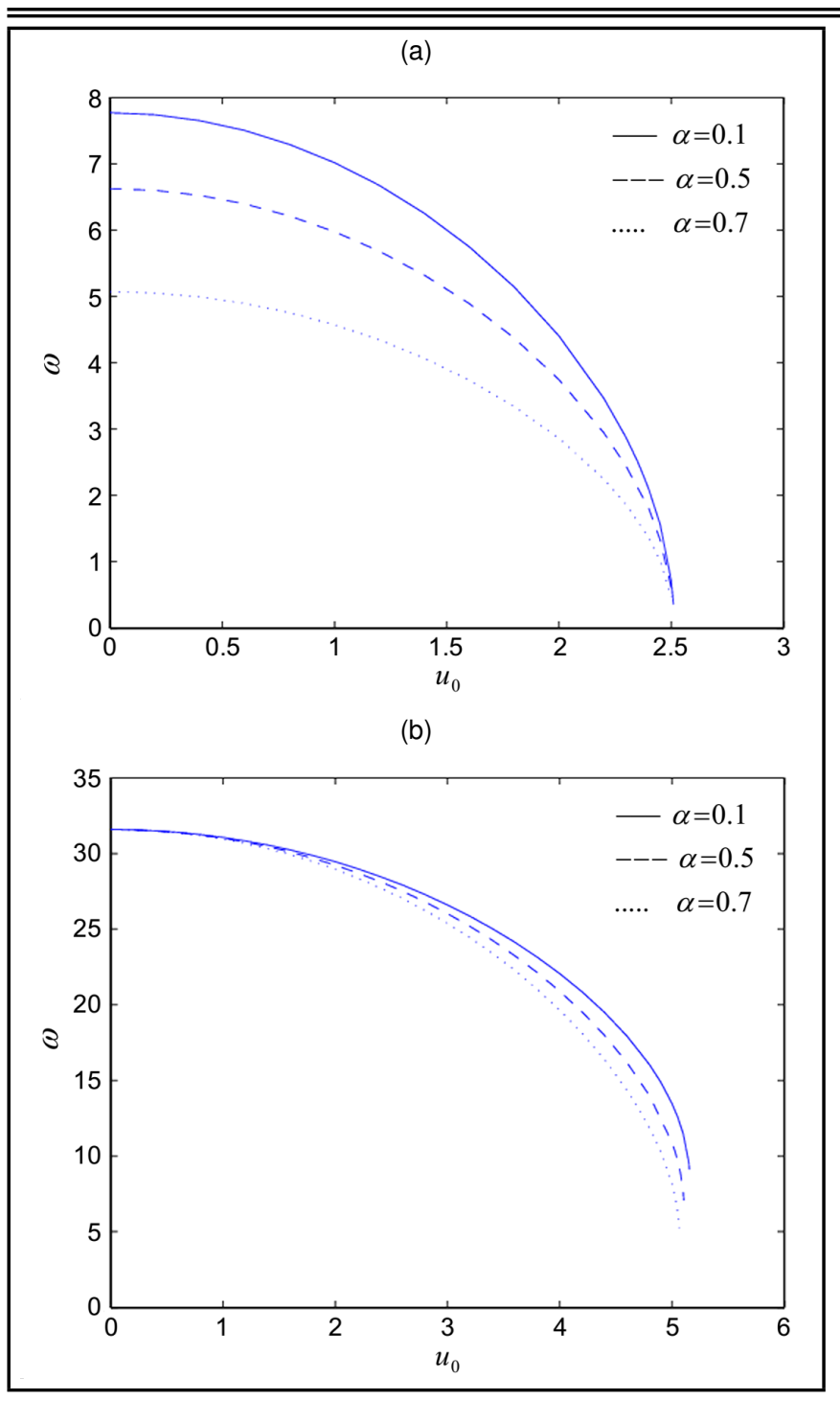

Figure 4. Axial velocity versus frequencies for different crack's depths $(\alpha)$; $\eta=0.5$. (a) first mode, (b) second mode.

In Fig. 6, axial velocity versus natural frequency has been drawn for multiple cracks ( $n$; one, five) when $\alpha_{p}=0.1$; $p=1 \ldots 5$. For case of one crack, it is in the middle of the beam. For case of multiple cracks, it is assumed that cracks are distributed with equal spans along the beam. From these figures, natural frequencies decrease with increasing number of cracks for both first and second modes. This is a result expected because the stiffness of the whole spring-beam system reduces in this case.

By assigning $v_{0}=0.5, v_{1}=0.1, \chi=1 / 10, v_{f}=0.8$ in Eq. (39), frequency-response curves have been drawn in Figs. 7 through 9. Because of being well-known phenomenon, trivial solutions have not plotted in these figures. Different control parameters have been compared in order to reach a good conclusion. In these figures, the nonlinearity effects bend the curves to the right causing multi-valued regions of the solutions. This is well-known phenomenon in the studies done on primary or internal resonances and is defined as hardening behavior of the system.

In Fig. 7, the frequency-response curves have been drawn for different crack depths $(\alpha ; 0.3,0.5)$ when crack position, $\eta=0.5$. As crack's depth $(\alpha)$ increases, multi-valued regions expand, and maximum amplitudes increase. Therefore, nonlin- (a)

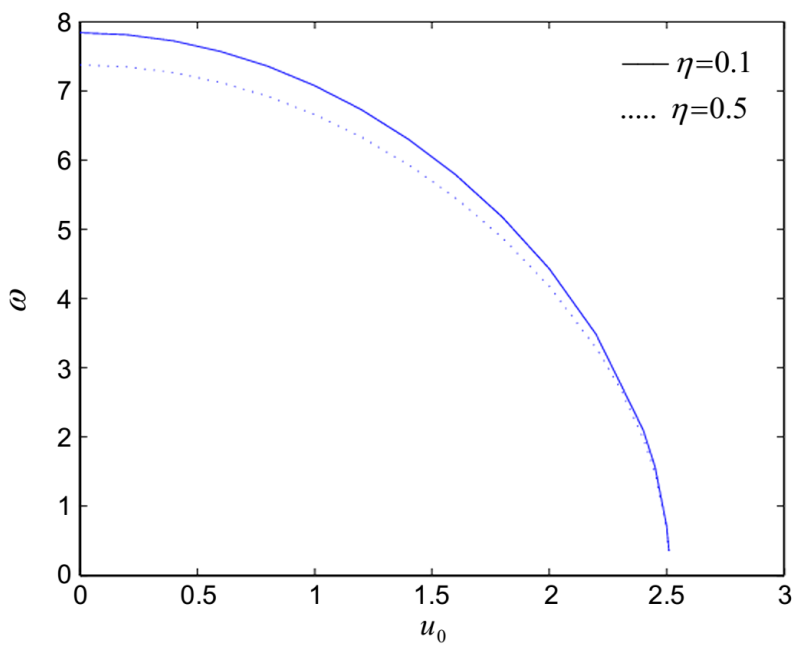

(b)

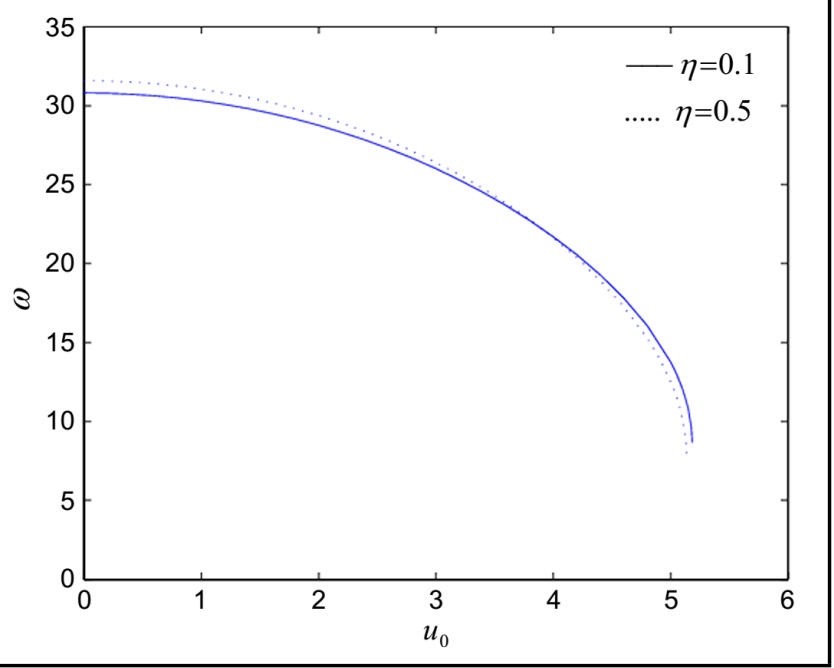

Figure 5. Axial velocity versus frequencies for different crack positions $(\eta)$; $\alpha=0.3$. (a) first mode, (b) second mode.

ear effects become more significant when the crack has more depth.

In Fig. 8, the frequency-response curves have been drawn for different crack's position, $(\eta ; 0.3,0.5)$ when $\alpha=0.3$. Maximum amplitudes decrease, and multi-valued regions narrow as the crack takes place at the middle point of the beam. Nonlinear effects are important when considering the beam part: including crack travels between the end support and middle point of the beam. Detuning parameter must be tuned to low values for this case.

In Fig. 9, the frequency-response curves have been drawn for different numbers of cracks, ( $n$; three, five) when $\alpha_{p}=$ 0.1 . As crack's number $(n)$ increases, maximum amplitudes increase, and multi-valued regions expand. In other saying, amplitudes increase with increasing beam's flexibility.

\section{CONCLUSIONS}

In this study, vibrations of axially moving beams with edge cracks were investigated. The cracks are distributed with equal spans throughout the beam's $x$ axis. For analysis of the problem, firstly, the beam has been divided into two parts in number according to crack tips, and then the parts connected with 


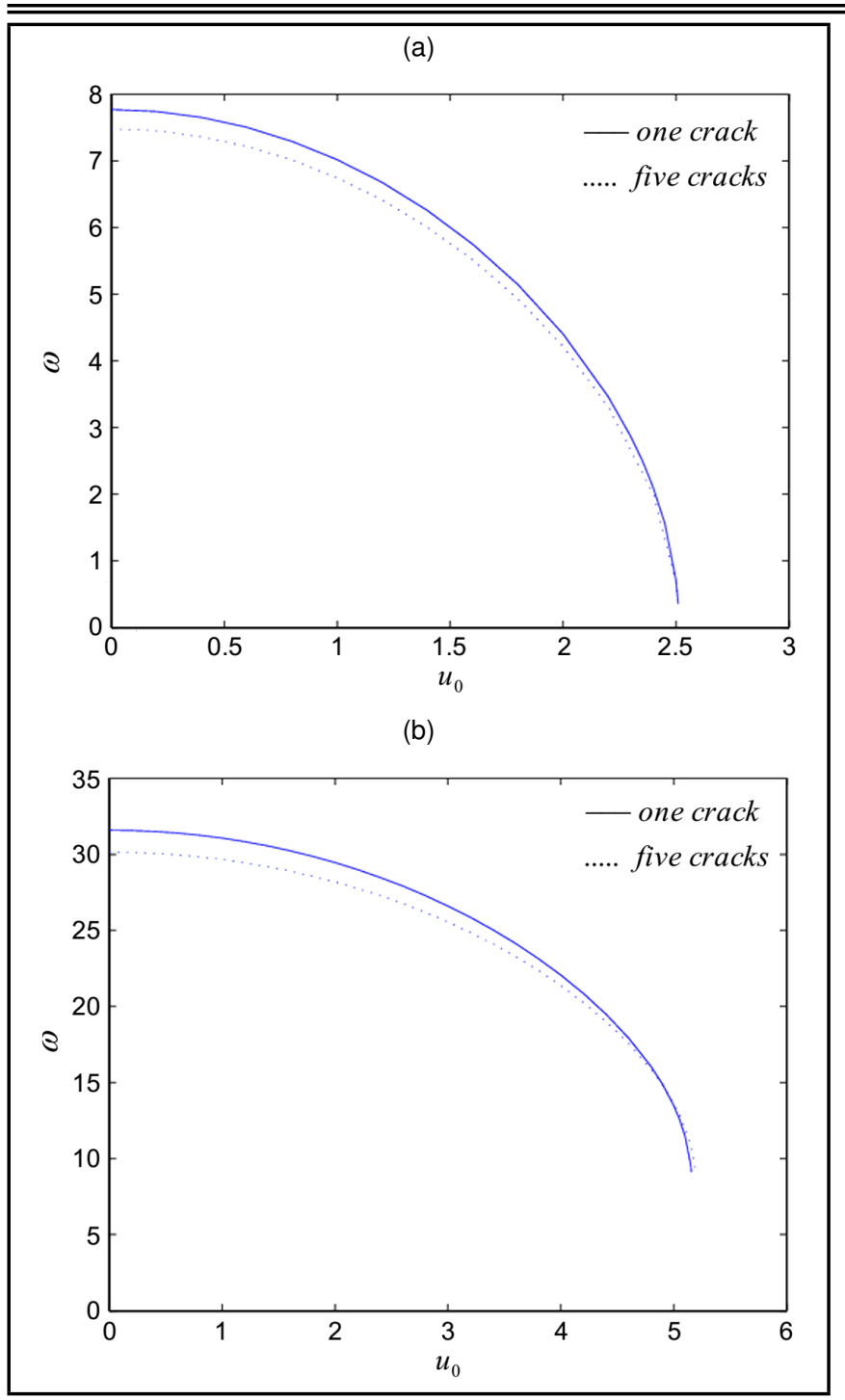

Figure 6. Axial velocity versus frequencies for different crack numbers; $\alpha_{p}=$ 0.1 . (a) first mode, (b) second mode.

each other by rotational and translational springs. Springs' stiffness has been obtained by means of Irwin's linear elastic fracture mechanics approach under static loading case and Castiglione's theorem. Secondly, for multiple cracks $n$, the beam has been divided into $n+1$ parts in number. For this beam-spring system, equations of motion and their corresponding conditions have been derived. Numerical solutions were searched under parametric resonance case. For axially moving beam with crack, investigations were based on these questions: 1) how natural frequencies would change with different control parameters; crack's depth, location as well as number, 2) and what kind of vibration behavior system exhibits under parametric resonance case.

Natural frequencies have been calculated according to crack depth, position parameters, as well as crack number on the beam. From figures, it was observed that frequencies of the first and second modes decrease with the increasing mean axial velocity. For the first mode, natural frequencies are at low values when the crack is up close to middle point of the beam. This case is different for the second mode because this mode is not symmetric for all velocity. The natural frequencies decrease with increasing crack depth. In case of travelling with high velocities, the effects of crack depth on natural frequen-

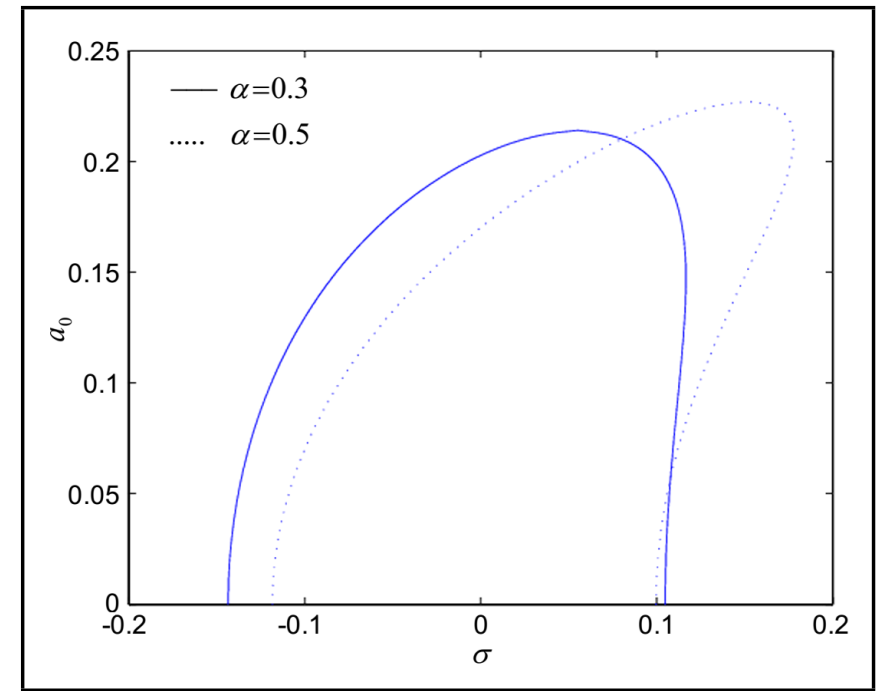

Figure 7. First mode force-amplitude curves for different crack depths $(\alpha)$; $\eta=0.5$.

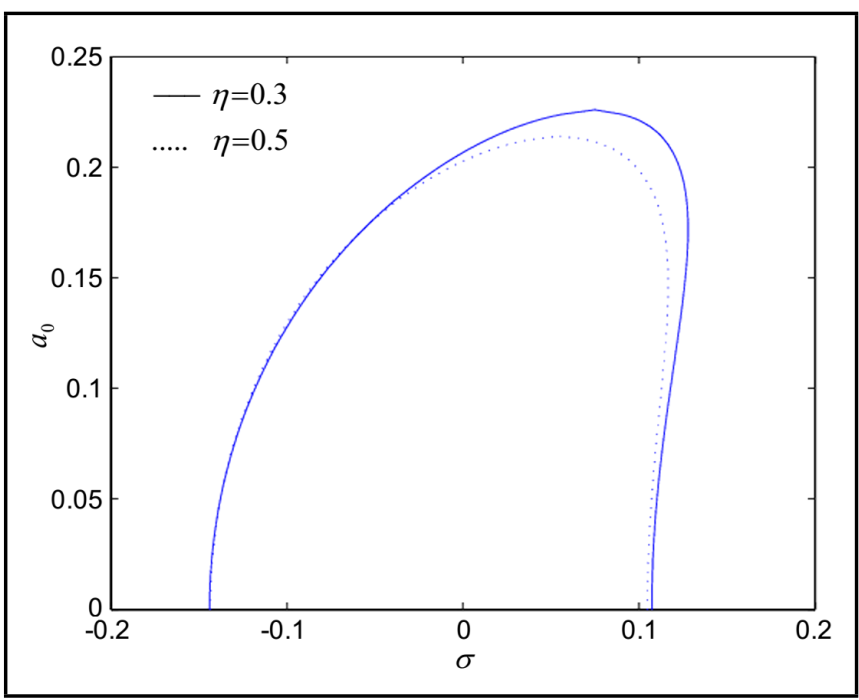

Figure 8. First mode force-amplitude curves for different crack positions $(\eta)$; $\alpha=0.3$.

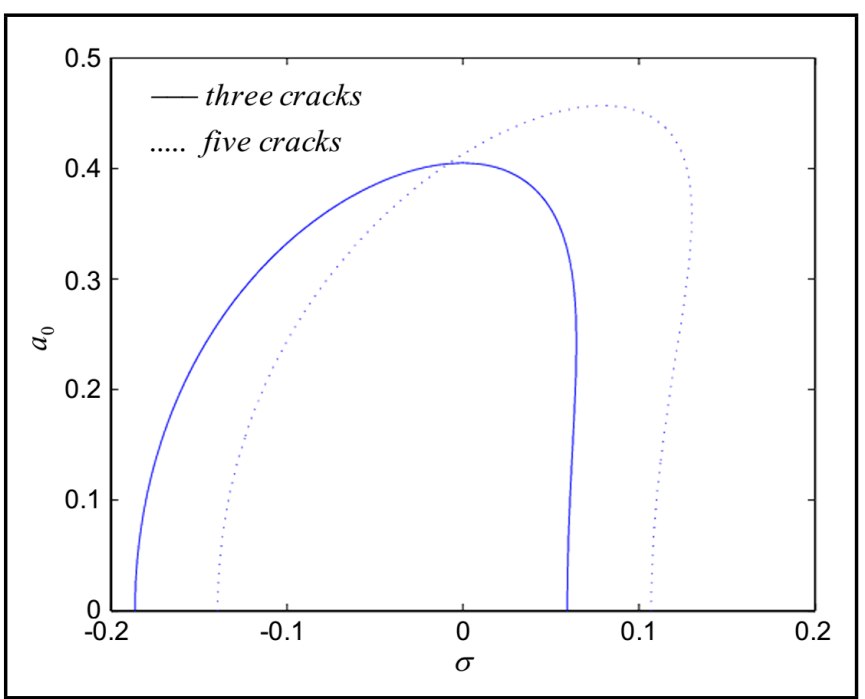

Figure 9. First mode force-amplitude curves for different crack numbers; $\alpha_{p}=0.1$. 
cies vanish. Beams with multiple cracks have low natural frequencies. For high velocities, there is no difference between one crack and multiple cracks in means of natural frequencies.

Vibration behavior of the system has been examined under steady state case during vibration. The system has hardening type behavior for an axially moving beam with edge cracks. When the beam part including cracks approaches the middle point of the beam, hardening behavior would lose its effects. Additionally, it could be concluded that the multi-valued region expands and maximum amplitude increases in the case of the crack depth being large and having multiple cracks. Detuning parameter must be tuned to the low values for these cases.

\section{REFERENCES}

1 Dimarogonas, A. D. Vibration of cracked structures: A state of the art review, Engineering Fracture Mechanics, 55 (5), 831-857, (1996). https://dx.doi.org/10.1016/00137944(94)00175-8

2 Sih, G. C., Ed. Method of fracture analysis and solutions of crack problems, Springer Netherlands, Leyden, (1973). https://dx.doi.org/10.1007/978-94-017-2260-5

$3 \mathrm{Wu}$, C. H. Maximum-energy-release-rate criterion applied to a tension-compression specimen with crack, Journal of Elasticity, 8 (3), (1978). https://dx.doi.org/10.1007/BF00130464

4 Tada, H., Paris, P. C., and Irwin, G. R. The stress analysis of cracks handbook, Paris Prod., St. Louis, (1985), 2nd Edition.

5 Anifantis, N. and Dimarogonas, A. Stability of columns with a single crack subjected to follower and vertical loads, International Journal of Solids and Structures, 19 (4), 281-291, (1983). https://dx.doi.org/10.1016/00207683(83)90027-6

6 Christides, S. and Barr, A. D. S. One dimensional theory of cracked Bernoulli-Euler beams, International Journal of Mechanical Sciences, 26, 639-648, (1984). https://dx.doi.org/10.1016/0020-7403(84)90017-1

7 Shen, M.-H. H. and Pierre, C. Free vibrations of beams with a single-edge crack, Journal of Sound and Vibration, 170 (2), 237-259, (1994). https://dx.doi.org/10.1006/jsvi.1994.1058

8 Chondros, T. G., Dimarogonas, A. D., and Yao, J. A continuous cracked beam vibration theory, Journal of Sound and Vibration, 215 (1), 17-34, (1998). https://dx.doi.org/10.1006/jsvi.1998.1640

9 Yang X. F., Swamidas A. S. J., and Seshadri R. Crack identification in vibrating beams using the energy method, Journal of Sound and Vibration, 244 (2), 339-357, (2001). https://dx.doi.org/10.1006/jsvi.2000.3498

10 Mazanoglu, K., Yesilyurt, I., and Sabuncu, M. Vibration analysis of multiple-cracked non-uniform beams, Journal of Sound and Vibration, 320, 977-989, (2009). https://dx.doi.org/10.1016/j.jsv.2008.09.010
11 Behzad, M., Meghdari, A., and Ebrahimi, A. A linear theory for bending stress-strain analysis of a beam with an edge crack, Engineering Fracture Mechanics, 75, 4695-4705 (2008). https://dx.doi.org/10.1016/j.engfracmech.2008.06.028

12 Ostachowicz, W. M. and Krawczuk, M. Analysis of the effect of cracks on the natural frequencies of a cantilever beam, Journal of Sound and Vibration, 150 (2), 191-201, (1991). https://dx.doi.org/10.1016/0022-460X(91)90615-Q

13 Sundermeyer, J. N. and Weaver, R. L. On crack identification and characterization in a beam by non-linear vibration analysis, Journal of Sound and Vibration, 183 (5), 857-871, (1995). https://dx.doi.org/10.1006/jsvi.1995.0290

14 Yokoyama, T. and Chen, M.-C. Vibration analysis of edge-cracked beams using a line-spring model, Engineering Fracture Mechanics, 59 (3), 403-409, (1998). https://dx.doi.org/10.1016/S0013-7944(97)80283-4

15 Wang, C.-S. and Lee, L.-T. Modified and simplified sectional flexibility of a cracked beam, Journal of Applied Mathematics, 2012, 543828, (2012). https://dx.doi.org/10.1155/2012/543828

16 Fernández-Sáez, J. and Navarro, C. Fundamental frequency of cracked beams in bending vibrations: An analytical approach, Journal of Sound and Vibration, 256 (1), 17-31, (2002). https://dx.doi.org/10.1006/jsvi.2001.4197

17 Mermertas, V. and Erol, H. Effect of mass attachment on the free vibration of cracked beam, The 8th International Congress on Sound and Vibration, Hong Kong, China, (2001).

18 Mendelshon, D. A., Vedachalam, S., Pecorari, C., and Mokashi, P. S. Nonlinear vibration of an edge-cracked beam with a cohesive zone, II: Perturbation analysis of Euler-Bernoulli beam vibration using a nonlinear spring for damage representation, Journal of Mechanics of Materials and Structures, 3 (8), 1589-1604, (2008). https://dx.doi.org/10.2140/jomms.2008.3.1589

19 Rao, D. S., Mallikarjuna Rao, K., and Raju, G. V. Crack identification on a beam by vibration measurement and wavelet analysis, International Journal of Engineering Science and Technology, 2 (5), 907-912, (2010).

20 Majkut, L. Acoustical diagnostics of cracks in beam like structures, Archives of Acoustics, 31 (1), 17-28, (2006).

21 Loya, J., López-Puente, J., Zaera, R., and Fernández-Sáez, J. Free transverse vibrations of cracked nanobeams using a nonlocal elasticity model, Journal of Applied Physics, 105, 044309, (2009). https://dx.doi.org/10.1063/1.3068370

22 Simpson, A. Transverse modes and frequencies of beams translating between fixed end supports, Journal of Mechanical Engineering Science, 15, 159-164, (1973). https://dx.doi.org/10.1243/JMES_JOUR_1973_015_031_02

23 Pasin, F. Ueber die stabilität der beigeschwingungen von in laengsrichtung periodisch hin und herbewegten stäben, Ingenieur-Archiv, 41, 387-393, (1972). 
24 Ponomareva, S. V. and van Horssen, W. T. On the transversal vibrations of an axially moving continuum with a timevarying velocity: Transient from string to beam behavior, Journal of Sound and Vibration, 325, 959-973, (2009). https://dx.doi.org/10.1016/j.jsv.2009.03.038

25 Wickert, J. A. and Mote, C. D. Classical vibration analysis of axially moving continua, Journal of Applied Mechanics, 57, 738-744, (1990). https://dx.doi.org/10.1115/1.2897085

26 Wickert, J. A. and Mote, C. D. Response and discretization methods for axially moving materials, Journal of Applied Mechanics, 44, 279-284, (1991). https://dx.doi.org/10.1115/1.3121365

27 Özkaya, E. and Pakdemirli, M. Vibrations of an axially accelerating beam with small flexural stiffness, Journal of Sound and Vibration, 234, 521-535, (2000). https://dx.doi.org/10.1006/jsvi.2000.2890

28 Pellicano, F. and Vestroni, F. Complex dynamics of high-speed axially moving systems, Journal of Sound and Vibration, 258 (1), 31-44, (2002). https://dx.doi.org/10.1006/jsvi.2002.5070

29 Öz, H. R., Pakdemirli, M., and Özkaya, E. Transition behavior from string to beam for an axially accelerating material, Journal of Sound and Vibration, 215 (3), 571-576, (1998). https://dx.doi.org/10.1006/jsvi.1998.1572

30 Sarigul, M. and Boyaci, H. Nonlinear vibrations of axially moving beams with multiple concentrated masses Part I: Primary resonance, Structural Engineering and Mechanics, 36 (2), 149-163, (2010). https://dx.doi.org/10.12989/sem.2010.36.2.149

31 Özkaya, E. and Öz, H. R. Determination of natural frequencies and stability region of axially moving beams using artificial neural networks method, Journal of Sound and Vibration, 252, 782-789, (2002). https://dx.doi.org/10.1006/jsvi.2001.3991

32 Öz H. R. and Pakdemirli, M. Vibrations of axially moving beam with time dependent velocity, Journal of Sound and Vibration, 227 (2), 239-257, (1999). https://dx.doi.org/10.1006/jsvi.1999.2247

33 Öz, H. R. Natural frequencies of axially travelling tensioned beams in contact with a stationary mass, Journal of Sound and Vibration, 259, 445-456, (2003). https://dx.doi.org/10.1006/jsvi.2002.5157
34 Lee, U. and Jang, I. On the boundary conditions for axially moving beams, Journal of Sound and Vibration, 306, 675690, (2007). https://dx.doi.org/10.1016/j.jsv.2007.06.039

35 Ding, H. and Chen, L.-Q. Galerkin methods for natural frequencies of high-speed axially moving beams, Journal of Sound and Vibration, 329, 3484-3494 (2010). https://dx.doi.org/10.1016/j.jsv.2010.03.005

36 Ding, H. and Chen, L.-Q. Natural frequencies of nonlinear vibration of axially moving beams, Nonlinear Dynamics, 63 (1-2), 125-134, (2011). https://dx.doi.org/10.1007/s11071-010-9790-7

37 Ghayesh, M. H., Kazemirad, S., and Amabili, M. Coupled longitudinal-transverse dynamics of an axially moving beam with an internal resonance, Mechanism and Machine Theory, 52, 18-34, (2012). https://dx.doi.org/10.1016/j.mechmachtheory.2012.01.008

38 Wang, L., Hu, Z., and Zhong, Z. Non-linear dynamical analysis for an axially moving beam with finite deformation, International Journal of Non-Linear Mechanics, 54, 5-21, (2013). https://dx.doi.org/10.1016/j.ijnonlinmec.2013.03.004

39 Ghayesh, M. H. and Amabili, M. Steady-state transverse response of an axially moving beam with time-dependent axial speed, International Journal of Non-Linear Mechanics, 49, 40-49, (2013). https://dx.doi.org/10.1016/j.ijnonlinmec.2012.08.003

40 Chen, L.-Q. and Yang, X.-D. Stability in parametric resonance of axially moving viscoelastic beams with time-dependent speed, Journal of Sound and Vibration, 284, 879-891, (2005). https://dx.doi.org/10.1016/j.jsv.2004.07.024

41 Mott, R. L. Applied strength of materials, CRC P., Boca Raton, (2007), 5th Edition.

42 Öz, H. R., Pakdemirli, M., and Boyaci, H. Nonlinear vibrations and stability of an axially moving beam with time-dependent velocity, International Journal of Non-Linear Mechanics, 36, 107-115, (2001). https://dx.doi.org/10.1016/S0020-7462(99)00090-6

43 Nayfeh, A. H. Introduction to perturbation techniques, Wiley, New York, (1981). 